\title{
A FULLY INTEGRATED MONOLITHIC LOCAL OSCILLATOR FOR LMDS RADIO LINK APPLICATIONS
}

\author{
M. Pagani, G. Favre, H. Andersson and M. Carminati \\ ERICSSON LAB ITALY
}

Via Cadorna, 73 - 20090 Vimodrone (MI) - Italy. maurizio.pagani@tei.ericsson.se

\begin{abstract}
This paper presents the design of a Local Oscillator for a new Broadband Access System based on Point to MultiPoint microwave Radio Links. The key advance is achieving good enough phase noise performance (100 dBc/ Hz@100KHz), over a relatively wide frequency band (15\%), with good pulling figure and harmonics suppression, at a low cost and small size, for volume production. A fully monolithic VCO/Prescaler solution, integrating the resonator, the reflection amplifier, a single-stage buffer amplifier, a divider by eight and all main bias circuitry into a $5.16 \mathrm{~mm}^{2} \mathrm{GaAs}$ chip has been developed. The development procedure set to make this possible, with specific emphasis to volume production issues, is hereby described. Some simulation and measured data are also reported. Moreover, some open issues and existing problems that still hinder first run design success are briefly discussed.
\end{abstract}

\section{INTRODUCTION}

MMIC technology is nowadays well recognized for its capability of reducing cost, size, weight and number of parts of modern microwave digital radios. To obtain extremely compact and cost effective microwave radio designs several kinds of MMIC chips and Multi-Chip Modules have been proposed [1]-[2], including power amplifiers, variable gain amplifiers, LNAs, Up/Down converters and multipliers.

The general trend is clearly to move from large area consuming hybrid and discrete component designs, usually requiring a significant tuning effort, to miniaturized MMIC based solutions, that can be automatically assembled and tested, with a significant reduction of cost and manufacturing cycle time. The latter is a big issue because short delivery time to customer and high manufacturing line throughput are becoming more and more important.

As well known, wireless communication systems market is continuously increasing at a quite high rate and new, fast growing, broadband multimedia services are even pushing more the microwave radio business. Cost effective, high performance design solutions and increased manufacturing capability are therefore mandatory to compete in such a challenging arena.

\section{LOW COST OSCILLATOR DESIGN ISSUE}

Microwave frequency source is commonly recognized as one of the most critical parts of all communication systems used in microwave links. Particularly, when dealing with digital modulation schemes, Local Oscillator phase noise is a real challenge for the radio design. It affects the received signals jitter, rapidly decreasing the Bit Error Ratio performance.

One solution commonly used in the past for microwave radios was a DRO (Dielectric Resonator Oscillator) design, able to achieve very low phase noise because of its high $\mathrm{Q}$ resonator. 
Unfortunately this kind of oscillator significantly increases the overall equipment cost for many reasons, the major one being the very reduced frequency tuning range they have. In order to cover the complete bandwidth it is therefore necessary to have a lot of different oscillators, typically one for each radio channel. Once the customer is able to decide the frequencies, the radio manufacturer must then select the appropriate DROs, assemble them into the radio units and test the system, before shipping it to the customer. That results in a significant lag in delivery time. The assembly and tuning procedure have also a great impact on the final cost..

An alternative solution is represented by the YIG (Yttrium Iron Garnet) oscillator, but today this is still a high cost and quite large component. Moreover it is extremely microphonic and that is a big limitation to its use in digital radio links because it increases BER (Bit Error Rate) or worst-case, it causes service link outages due to the modem losing lock.

Another possibility is to use a CRO (Coaxial Resonator Oscillator) design. In this case it is usually used a silicon transistor and a compact high $\varepsilon_{\mathrm{r}}$ coaxial resonator typically operated in the 1 to $3 \mathrm{GHz}$ frequency range. This kind of solution allows the use of SMD (Surface-Mount Devices) assembly technology and a relative low cost automatic building process. Unfortunately, so far, it is still difficult to design discrete $\mathrm{CRO}$ at higher frequency than few $\mathrm{GHz}$ and, as consequence, it is necessary to provide a long chain of frequency multipliers, if the final frequency is in the millimeter-wave band.

\section{OUR PROPOSED SOLUTION}

Here we present a new solution for a Local Oscillator design featuring low phase noise performance, low cost and extremely reduced size. The only way to realize such an oscillator is to use HBT (Heterojunction Bipolar Transistor) MMIC technology. Some MMIC oscillators have been already reported in literature [3], but all of them have the resonator, or at least the varactor element outside the MMIC itself. Our design is a fully integrated wideband VCO followed by a buffer amplifier as shown in fig.1, with also a divider by eight prescaler hosted into the same chip.

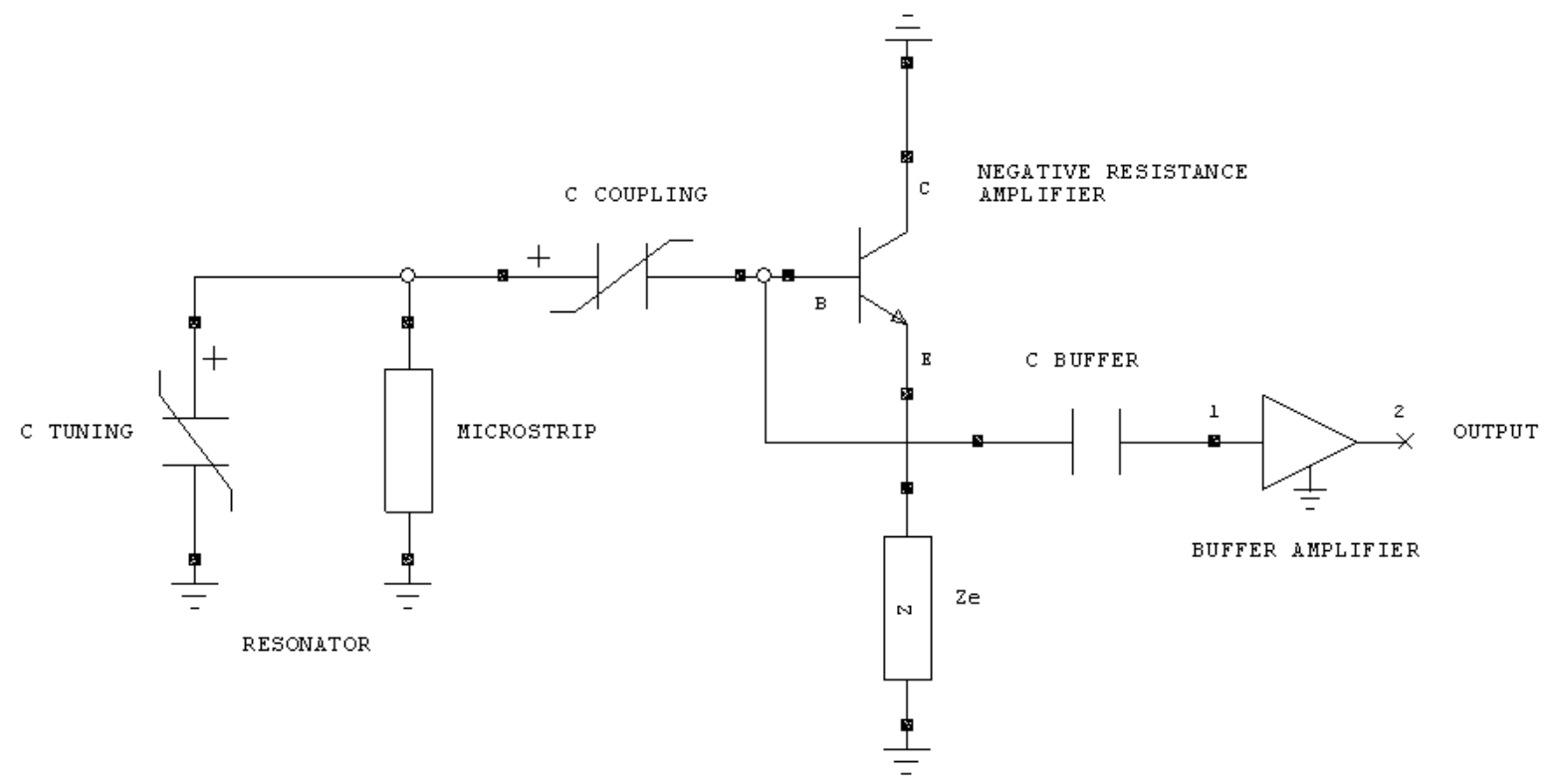

Fig. 1. - Simplified sketch of the negative resistance VCO. 
The single-stage buffer amplifier is cascaded to the VCO in order to reduce loading, pushing and pulling effects, providing at the same time some harmonics filtering and enough gain to increase the output power. A particular care has been posed in its design to obtain flat in-band gain, proper out-band attenuation and high reverse isolation. In such a way, harmonics and spurious signals, causing mixing products that often generate interfering radio emissions, are particularly counteracted.

This Monolithic VCO design has demonstrated a good process-tolerance and has been easily integrated into an MCM (Multi Chip Module) that has proven to be very suitable for automatic testing and high volume production.

\section{DEVELOPMENT PROCEDURE FOR THE LOW PHASE NOISE MMIC VCO.}

The combination of using a low-noise device and a high $\mathrm{Q}$ resonator is effective for creating a low phase noise oscillator. It is well known that, due to their vertical device structure, HBTs exibit much better low frequency noise characteristics than FET-type devices. Consequently, with HBT technology it has been possible to achieve state-of-the-art low phase noise VCOs at microwave frequencies. By using GaAs substrate has also been possible to keep the resonator on chip because of its lower loss if compared with Si.

A careful study of the coupling effects between the resonator and the negative resistance amplifier is the key point of our development procedure. The simplified oscillator scheme is shown in fig.1. From the picture, can be noted that it is possible to easily change the coupling factor between the reflection amplifier and the resonator. This is really useful because of the extreme wideband of this VCO. Without changing the coupling factor it would be difficult to keep the same Q value over the complete tuning range. In this way it is also possible to experimentally find out the best coupling factor in order to achieve the lowest phase noise. Of course it is not possible to reduce the coupling below a certain value that can be experimentally investigated because the circuit would stop to oscillate

The design has been firstly carried out with the classical approach described in literature by Kurokawa. Both linear and non linear simulations have been performed using a commercial CAD tool. Linear simulation is useful to evaluate resonator characteristics versus $\mathrm{C}$ Coupling variation and find out the gain margin, at each operating condition.

Particular care was posed in the reflection amplifier design in order to avoid spurious oscillation outside the band of interest.. By means of Harmonic Balance technique is possible to predict the harmonics content and phase noise performance. Simulation results at one operating condition are shown in fig.2 and fig. 3. Measured data of a typical sample are shown in fig. 4 and fig.5.

A pretty good agreement can be noted. That is also because the device model was carefully tailored to the specific transistor configuration used in the current design, during a previous development phase. Changing of the transistor configuration would require a new modeling effort because global nonlinear noise model of microwave device is still an open issue that should be investigated more deeply by the microwave research community.

A phase noise of $100 \mathrm{dBc} / \mathrm{Hz}$ at $100 \mathrm{KHz}$ has been measured over a $15 \%$ bandwidth at a frequency around $7 \mathrm{GHz}$. A harmonics suppression higher than $20 \mathrm{dBc}$ has been achieved. 


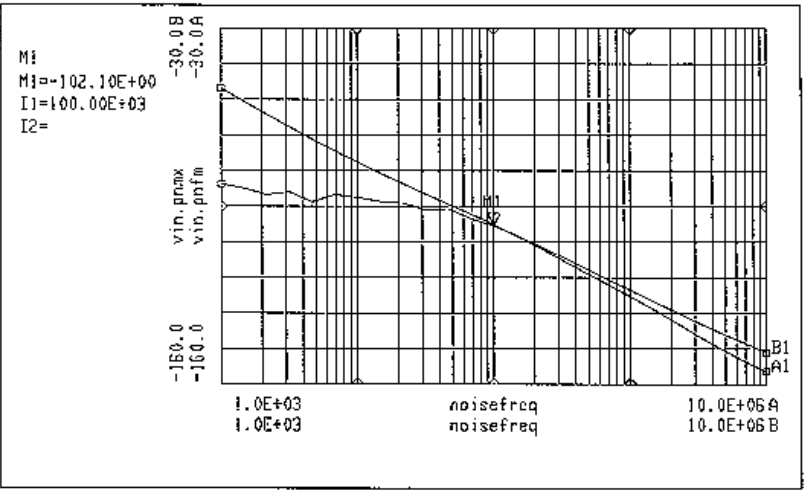

Fig. 2 - Simulated phase noise.

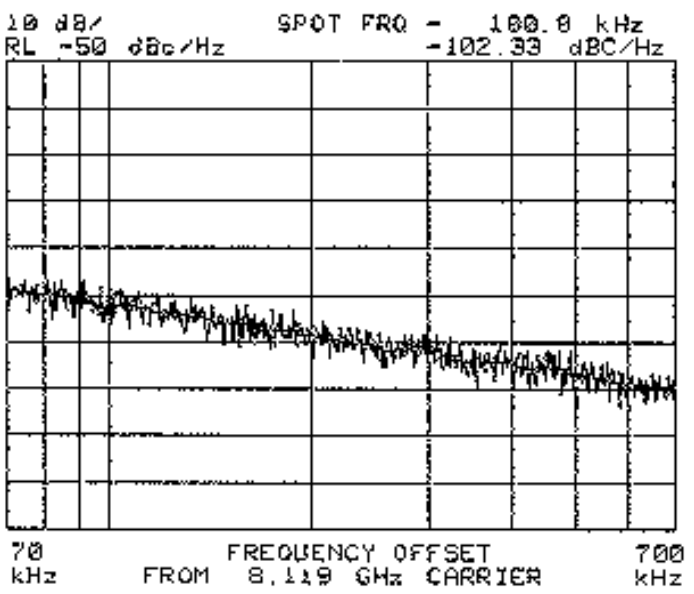

Fig. 4 - Measured phase noise.

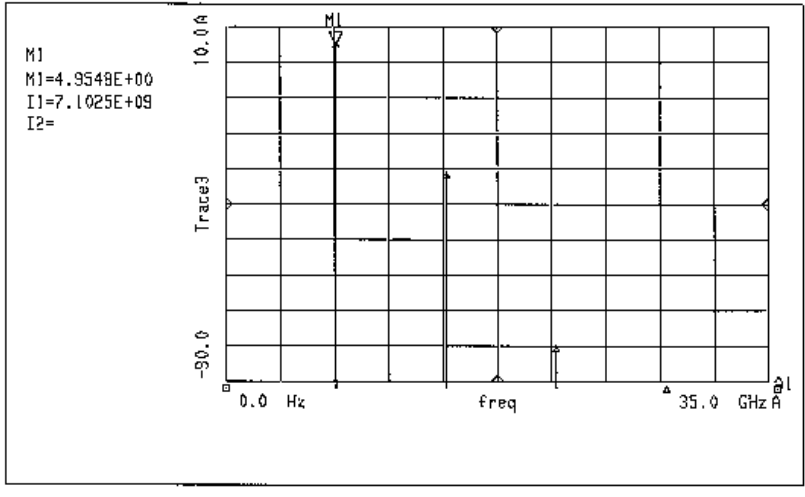

Fig. 3 - Simulated output spectrum.

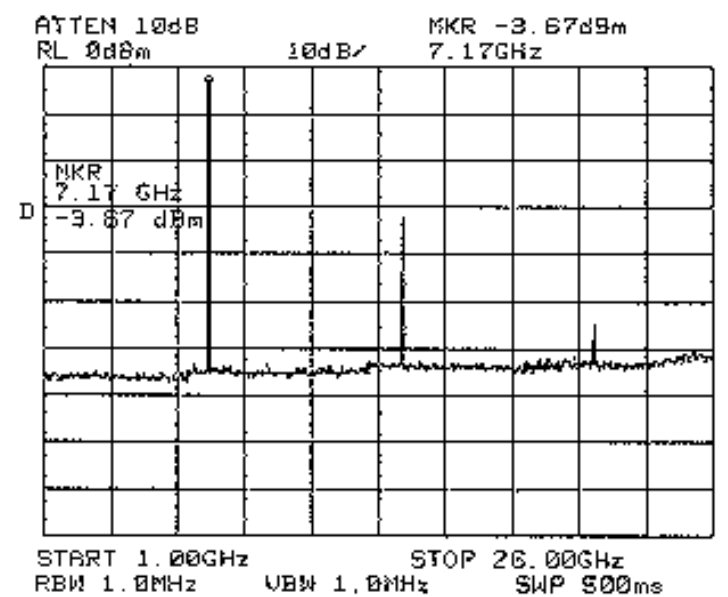

Fig. 5 - Measured output spectrum.

\section{CONCLUSION}

The first, at our knowledge, fully integrated monolithic Local Oscillator including the resonator, the reflection amplifier, a buffer amplifier, a divider by eight and all bias circuitry into a $5.16 \mathrm{~mm}^{2}$ GaAs chip has been developed. The development procedure, with specific emphasis to volume production issues has been described. Some simulated and measured data have also been reported.

\section{AKNOWLEDGEMENT}

This work has been made possible only thanks to the valuable contribution of our colleagues of Ericsson EMW- Gothenburg, particularly Mats, Neil, Magnus, Jan and Erik.

The authors would like to aknowledge all of them and Gregorio for their help.

\section{REFERENCES}

[1] M. Pagani, M. Remonti, S. Velati and A. Panzeri " GaAs MMICs for cost effective insertion in digital radio link transceivers”. Proc. GAAS'97 , Bologna, Italy, October 1997.

[2] B. Davide, M. Pagani and A. Panzeri "Modular Low Cost, High efficiency, C Band Power Amplifiers for SDH radio", Proc. of $5^{\text {th }}$ European Conf. On Fixed Radio Systems and Networks, Bologna, Italy, May1996.

[3] H. Suwaki, T. Nakagawa, T. Ohira "An MMIC Local Oscillator for 16-QAM Digital Microwave Radio Systems”, IEEE Trans. Microwave Theory Tech., vol. 43, no. 6, pp. 1230-1235, June 1995. 